\title{
The impact of film thickness and substrate surface roughness on the thermal resistance of aluminum nitride nucleation layers
}

\author{
Zonghui Su, ${ }^{1}$ Justin P. Freedman, ${ }^{2}$ Jacob H. Leach, ${ }^{3}$ Edward A. Preble, ${ }^{3}$ Robert F. Davis, ${ }^{2}$ \\ and Jonathan A. Malen ${ }^{1,2, a)}$ \\ ${ }^{1}$ Department of Mechanical Engineering, Carnegie Mellon University, Pittsburgh, Pennsylvania 15213, USA \\ ${ }^{2}$ Department of Materials Science and Engineering, Carnegie Mellon University, Pittsburgh, \\ Pennsylvania 15213, USA \\ ${ }^{3}$ Kyma Technologies, Raleigh, North Carolina 27617, USA
}

(Received 20 March 2013; accepted 14 May 2013; published online 3 June 2013)

\begin{abstract}
Thickness dependent thermal conductivity measurements were made on aluminum nitride (AlN) thin films grown by two methods on the (0001) surfaces of silicon carbide ( $\mathrm{SiC}$ ) and sapphire substrates with differing surface roughness. We find that the AlN itself makes a small contribution to the overall thermal resistance. Instead, the thermal boundary resistance (TBR) of $5.1 \pm 2.8 \mathrm{~m}^{2} \mathrm{~K} / \mathrm{GW}$ between the AlN and substrate is equivalent to $240 \mathrm{~nm}$ of highly dislocated AlN or $1450 \mathrm{~nm}$ of single crystal AlN. An order-of-magnitude larger TBR was measured between AlN films and SiC substrates with increased surface roughness ( $1.2 \mathrm{~nm}$ vs. $0.2 \mathrm{~nm}$ RMS). Atomic resolution TEM images reveal near-interface planar defects in the AlN films grown on the rough $\mathrm{SiC}$ that we hypothesize are the source of increased TBR. (C) 2013 AIP Publishing LLC. [http://dx.doi.org/10.1063/1.4808238]
\end{abstract}

Nitride semiconductors are essential for blue/green light emitting diodes (LEDs) and high electron mobility transistors (HEMTs). LEDs are now being pushed to high power for lighting applications causing considerable heat generation due to Joule heating and inefficiencies in light production. ${ }^{1}$ HEMTs are essential to high-power and high-speed switching operations that generate heat as a byproduct., ${ }^{2,3}$ While thermal packaging is essential to minimize operating temperatures, nearly half of the total thermal resistance comes from the nitride device itself. ${ }^{2,3}$ Experiments on bulk nitrides show that thermal transport is phonon-dominated. ${ }^{4-7}$ Internal thermal resistance of nitride devices is complicated by the presence of interfaces between films and defects within films that scatter phonons and suppress thermal conductivity below bulk values.

Non-native silicon carbide $(\mathrm{SiC})$ and sapphire substrates are used for the commercial growth of nitride films because gallium nitride $(\mathrm{GaN})$ and aluminum nitride (AlN) substrates are not yet economically viable. Growth is initiated with an AlN nucleation layer because direct growth of $\mathrm{GaN}$ on these substrates is not favorable at high temperatures. Due to a mismatch in the lattice parameters $(a)$ of $\operatorname{AIN~}\left(a_{A I N}=3.07 \AA\right)$ with $\operatorname{SiC}\left(a_{S i C}=3.11 \AA\right)$ and sapphire $\left(a_{S a p p}=4.785 \AA\right)$, dislocations and surface defects form at the interface and impact the quality of subsequent growth. Though $\mathrm{SiC}$ and AlN have a small mismatch (1\%), their high elastic moduli require stress relief through such defect formation. ${ }^{8,9}$

Prior studies agree that the AlN nucleation layer is a dominant thermal resistance in both $\mathrm{LED}^{10}$ and HEMT architectures. ${ }^{2,3,11,12}$ The source of this thermal resistance, however, is as yet experimentally unresolved as the total thermal resistance $R_{T}$ consists of three components: (1) the AlN/substrate TBR (TBR $\left.R_{\text {sub }}\right)$, (2) the AlN intrinsic resistance $L_{\text {AIN }} / k_{\text {AlN }}$

\footnotetext{
a) Author to whom correspondence should be addressed. Electronic mail: jonmalen@andrew.cmu.edu.
}

(where $L$ is film thickness and $k$ is thermal conductivity), and (3) the GaN/AlN TBR. Our prior study suggests that $T B R_{\text {sub }}$ is largest for AIN films grown on mechanically polished (MP) $\mathrm{SiC}$ substrates. ${ }^{5}$ Nonetheless, it is unclear whether this conclusion holds for $\mathrm{SiC}$ vs. sapphire substrates, different growth techniques, or varied surface roughness of the substrate that is known to affect TBR in other systems. ${ }^{13,14}$

To understand and isolate these effects we herein report a study of thermal resistance as a function of film thickness for AlN films grown by organometallic vapor phase epitaxy (OMVPE) and plasma vapor deposition (PVD) on $\mathrm{SiC}$ and sapphire substrates of varying roughness. Thermal conductivity measurements were made using the 3 -omega method as well as frequency domain thermoreflectance (FDTR), which are established approaches for accurate measurements of cross plane thermal conductivity in thin films. ${ }^{15-17}$

AlN growth details can be found in the supplementary online material. ${ }^{18}$ Immediately prior to AlN growth, the $\mathrm{SiC}$ and sapphire substrates were ultrasonically cleaned in solvents and the $\mathrm{SiC}$ substrates were then immersed in $\mathrm{HF}$ to remove the native oxide. AlN films of thickness $50 \mathrm{~nm}$, $100 \mathrm{~nm}, 250 \mathrm{~nm}$, and $500 \mathrm{~nm}$ were grown by OMVPE on MP $6 \mathrm{H} \mathrm{SiC} \mathrm{(0001)} \mathrm{(MP-SiC)} \mathrm{and} \mathrm{chemomechanically} \mathrm{polished}$ (CMP) 4H SiC (0001) (CMP-SiC) substrates. Additionally, AlN films of thickness $50 \mathrm{~nm}, 100 \mathrm{~nm}, 250 \mathrm{~nm}$, and $500 \mathrm{~nm}$ were grown by PVD on CMP-SiC and sapphire substrates. Substrate RMS roughness was characterized by tapping mode AFM and film thicknesses were measured by spectral reflectance with an accuracy of $\pm 1 \mathrm{~nm}$. Transmission electron microscopy (TEM) imaging of the AlN/SiC interface and the (0001) AlN surface for determination of dislocation density followed previously reported procedures ${ }^{10}$ summarized in the supplementary online material. ${ }^{18}$

To measure thermal conductivity via the 3-omega method one uses a resistive heater/thermometer that is microfabricated on the sample surface to induce and measure a periodic 
temperature change that has a sample-dependent amplitude. The experimental design of the 3-omega system is shown in Figure 1(a) where a schematic of the four pad 3-omega pattern is boxed in a dashed line. The nominal dimensions of the $\mathrm{Au}$ heater were $1500 \mu \mathrm{m}$ in length, $10 \mu \mathrm{m}$ in width, and $250 \mathrm{~nm}$ in thickness (including a $5 \mathrm{~nm} \mathrm{Ti}$ adhesion layer), though the actual widths varied and were individually measured. The 3-omega method demands an electrically insulating sample. Though AlN is an electrical insulator, pinholes in the thin films necessitated the use of a $70 \mathrm{~nm}$ sputtered $\mathrm{SiO}_{2}$ layer on the semiconducting MP-SiC substrate. The CMP-SiC was undoped, but measurements with and without $\mathrm{SiO}_{2}$ were made for comparison. The effective thermal conductivities of the AlN films $\left(k_{\mathrm{AlN} \text {-eff }}\right)$ were determined by fitting the temperature amplitude as a function of heating frequency to an analytical heat conduction model. ${ }^{17}$ Reported uncertainties in $k_{\text {AlN-eff }}$ result from uncertainty in the heater geometry, film thickness, and heater coefficient of thermal resistance. Further details can be found in the supplementary online material. ${ }^{10,15,18}$

Representative temperature amplitude vs. frequency data and analytical fits are shown in Figure 1(b) for AlN films grown by OMVPE on CMP SiC. The temperature amplitude can be broken down as $\Delta T(\omega)=\Delta T_{\text {sub }}(\omega)+\Delta T_{A l N}$, where $\Delta T_{\text {sub }}(\omega)$ is the portion due to thermal resistance in the substrate. The frequency independent temperature amplitude due to the AlN film is defined as $\Delta T_{A I N}=\left(L_{A I N} /\right.$ $\left.k_{A l N-\text { eff }}\right)\left(I^{2} R_{e} / l w\right)$, where $k_{A l N \text {-eff }}$ includes contributions from the TBRs of the bounding interfaces, $R_{e}$ is the heater's electrical resistance, $l$ is the heater length, and $w$ is the heater width. ${ }^{17}$ The lowest line (red) in Figure 1(b) represents $\Delta T_{\text {sub }}(\omega)$. The offset between the red line and the data is $T_{A l N}$. This offset increases with film thickness, which directly indicates that the AlN thermal resistance $R_{T}=L_{\text {AlN }} / k_{\text {AlN-eff }}$ increases with film thickness.

Measured values of $k_{\mathrm{AlN} \text {-eff }}$ are plotted as a function of film thickness in Figure 2(a). For all films, $k_{\text {AlN-eff }}$ increases with increasing film thickness. Independent of thickness, AlN films grown on MP SiC substrates have $k_{A I N \text {-eff }}$ values that are one order-of-magnitude lower than AlN films grown on the other substrates. AlN films grown on CMP SiC and sapphire substrates, by both OMVPE and PVD, have similar thermal conductivities at a given thickness. Although the MP-SiC is $6 \mathrm{H}$, and the CMP-SiC is $4 \mathrm{H}$, the polytype should not strongly influence growth on the (0001) surface. Instead, we believe that the key difference between the MP-SiC and the other substrates is surface roughness, which is listed in Table I. The root mean square (RMS) roughness of the MP$\mathrm{SiC}$ substrate is six times larger than the RMS roughness of the CMP-SiC or sapphire substrates. The presence of the $\mathrm{SiO}_{2}$ layer cannot be the cause of the drastic difference between MP-SiC and CMP-SiC based films because the $\mathrm{SiO}_{2}$ layer causes only a two-fold reduction in $k_{\text {AlN-eff }}$ for $\mathrm{SiO}_{2} / \mathrm{OMVPE}-\mathrm{AlN} / \mathrm{CMP}-\mathrm{SiC}$ vs. Ti/OMVPE-AlN/CMP$\mathrm{SiC}$ - an effect attributed to differences in the Ti/AlN and $\mathrm{SiO}_{2} / \mathrm{AlN}$ TBRs. Hence, the roughness effect is dominant in differentiating our data; it is more important than either the AlN/substrate lattice mismatch or the AlN growth technique.

Atomic resolution TEM images of the $\mathrm{AlN} / \mathrm{SiC}$ interface, shown in Figure 3, reveal microstructural differences between AlN films grown on MP-SiC and CMP-SiC substrates. In both images, the $\mathrm{SiC}$ is on the left and the AIN is on the right of the interface. The AIN/CMP-SiC interface shown in Figure 3(a) is clearly defined with no apparent strain in the film (i.e., beyond the strain due to the inherent lattice mismatch of SiC and AlN). In contrast, the AlN/MP-SiC interface shown in Figure 3(b) indicates that the marked microstructural roughness of the (0001) surface of the MP-SiC wafer generated stresses and resultant strains that produced planar defects within the first few atomic layers in the AIN film. As the periodicity of the AIN is recovered within nanometers of the interface for both samples, we conclude that defects at the interface impact the TBR of the heterostructures. The densities of threading
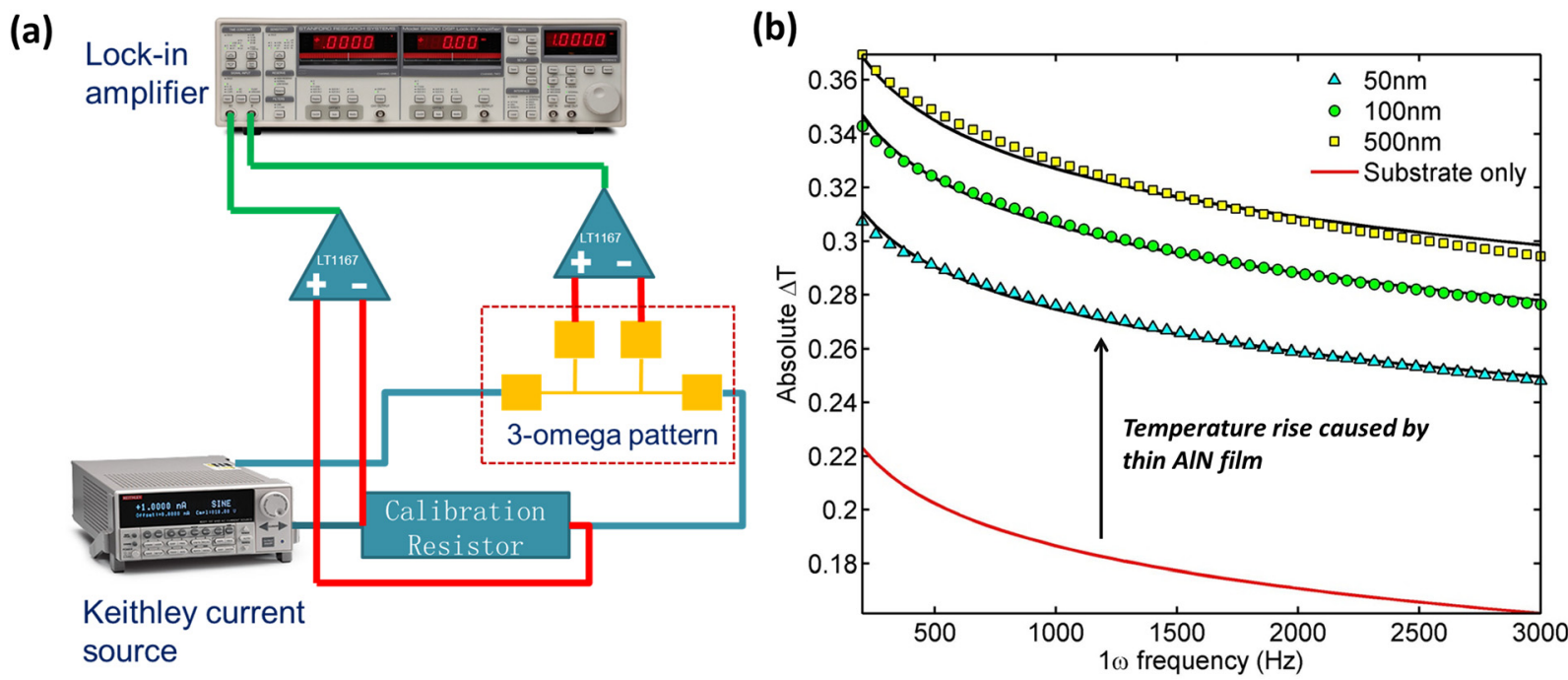

FIG. 1. Experimental setup and raw data. (a) The 3-omega method experimental setup uses a Au 3-omega pattern that is microfabricated on the sample surface as a resistive heater/thermometer. A current source at a frequency 1 drives periodic heating in the heater/thermometer, while a lock-in amplifier senses the sample-dependent thermal response through a circuit designed to reject common-mode noise. (b) The amplitude of the temperature response vs. the modulation frequency for OMVPE AlN films of varying thickness on the CMP SiC substrate. The offset from the red line represents the induced temperature change due to the AlN film and is correlated with film thickness. 

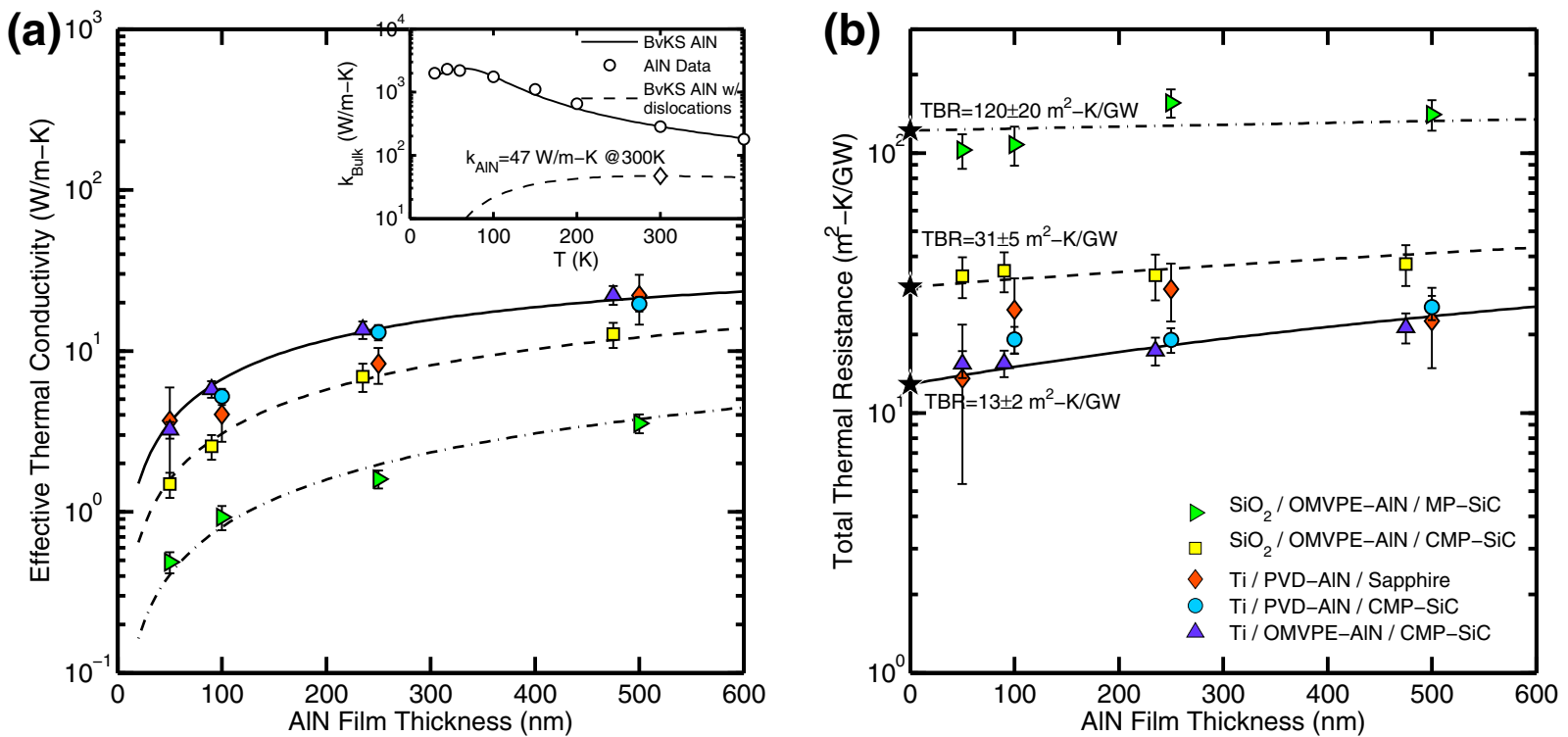

FIG. 2. (a)Thermal conductivity versus AlN film thickness on different substrates. The AlN films grown on MP SiC substrates have the lowest thermal conductivities over all the thicknesses. The thermal conductivity differences between films grown on MP vs. CMP SiC substrates of differing roughness are more significant than differences due to growth method or substrate material. (a-inset) The Born von Karman Slack (BvKS) model for thermal conductivity accurately matches bulk experimental data from Ref. 5 , and predicts $k_{\mathrm{AlN}}=47 \mathrm{~W} / \mathrm{mK}$ at $T=300 \mathrm{~K}$ when phonon dislocation scattering based on Ref. 10 is considered. (b) Comparison of AlN films' total thermal resistances versus film thickness. This plot clarifies that the primary thermal resistance comes from the thermal boundary resistance, rather than the film itself. Solid line fits in (a) and (b) determine values of TBR based on Eq. (1) with $k_{\text {AIN }}=47 \mathrm{~W} / \mathrm{mK}$.

dislocations, measured in plan view at the top of the $100 \mathrm{~nm}$ AlN films were of similar order $\left(4 \pm 1 \times 10^{10} \mathrm{~cm}^{-2}\right)$ for the AlN films grown on MP-SiC and CMP-SiC. These results further confirm that the discrepancy in $k_{A l N-e f f}$ comes from TBR and not the intrinsic thermal conductivity of the AlN.

Thermal boundary resistance plays a significant role in explaining our effective thermal conductivity trends. To clarify this point, we plot $R_{T}=L_{A l N} / k_{A I N-e f f}$ vs. $L_{A I N}$ in Figure 2(b). This shows that $R_{T}$ is only weakly dependent on film thickness. In the simplest terms, $R_{T}$ can be decomposed into three series resistances as

$$
R_{T}=L_{A l N} / k_{A l N}+T B R_{\text {sub }}+T B R_{\text {top }} .
$$

The intrinsic thermal conductivity of AlN $\left(k_{A l N}\right)$ was calculated using the Born von Karman Slack (BvKS) model that uses the Born von Karman dispersion relationship and scattering based on the modified Callaway model. ${ }^{19,20}$ The BvKS model defines the frequency dependent phonon mean free path $\Lambda_{i}(\omega)$ due to defects and Umklapp scattering as $\Lambda_{i}=v_{g}\left(A \omega^{4}+P \omega^{2} T e^{-C_{U} / T}\right)^{-1}$, where $v_{g}$ is the phonon group velocity, $A$ is a material dependent constant that defines the defect scattering rates, and $P$ and $C_{U}$ are material dependent constants that define the Umklapp scattering rates. The constants were determined from the best fit to single

TABLE I. Surface roughness and lattice parameter of different substrates.

\begin{tabular}{lcc}
\hline \hline Material & Roughness $(\mathrm{nm})$ & Lattice parameter $(\AA)$ \\
\hline MP SiC & 1.2 & 3.11 \\
CMP SiC & 0.2 & 3.11 \\
Sapphire & 0.2 & 4.785 \\
AlN & - & 3.07 \\
\hline \hline
\end{tabular}

crystal AlN thermal conductivity vs. temperature data, ${ }^{5}$ shown in the Figure 2(a) inset, as $A=9.5 \times 10^{-45} \mathrm{~s}^{3}$, $P=2.1 \times 10^{-19} \mathrm{sK}^{-1}$, and $C_{U}=390 \mathrm{~K}$. The addition of dislocation scattering based on Su et al. ${ }^{10}$ with an areal density of $4 \times 10^{10} \mathrm{~cm}^{-2}$ results in the dashed line in the Figure 2(a) inset and a prediction of $k_{\mathrm{AlN}}=47 \mathrm{~W} / \mathrm{mK}$ at $T=300 \mathrm{~K}$ for our films.

Based on Eq. (1) and the prediction of $k_{\mathrm{AlN}}$, a fit (see black lines in Figure 2(b)) to our $R_{\mathrm{T}}$ data was extrapolated to $L=0$ to determine the summed $T B R$ due to both interfaces $\left(T B R_{\text {sub }}+T B R_{\text {top }}\right)$ as $120 \pm 20 \mathrm{~m}^{2} \mathrm{~K} / \mathrm{GW}$ for $\mathrm{SiO}_{2} / \mathrm{AlN} / \mathrm{MP}-$ $\mathrm{SiC}, 31 \pm 5 \mathrm{~m}^{2} \mathrm{~K} / \mathrm{GW}$ for $\mathrm{SiO}_{2} / \mathrm{AlN} / \mathrm{CMP}-\mathrm{SiC}$, and $13 \pm 2$ $\mathrm{m}^{2} \mathrm{~K} / \mathrm{GW}$ for the other three samples. Through substitution of the relation $R_{T}=L_{A I N} / k_{A l N-e f f}$ into Eq. (1), we were also able to plot the predicted $k_{\mathrm{AlN} \text {-eff }}$ as black lines in Figure 2(a). Equation (1) accurately fits our data, though for thinner or less defective AlN films, it may be necessary to consider ballistic phonon transport based on the Boltzmann Transport Equation. $^{21-23}$

$T B R_{\text {sub }}$ is now isolated from $T B R_{\text {top. }}$ Quantitatively, the three values of $T B R_{\text {sub }}+T B R_{\text {top }}$ listed in Table II result from combinations of the following four interfaces: AlN/CMP$\mathrm{SiC}, \mathrm{AlN} / \mathrm{MP}-\mathrm{SiC}, \mathrm{Ti} / \mathrm{AlN}$, and $\mathrm{SiO}_{2} / \mathrm{AlN}$. Hence, we have three equations and four unknowns that can be solved if one unknown is eliminated. We eliminated $T B R_{\mathrm{Ti} / \mathrm{AlN}}$ using a direct measurement by FDTR. This measurement technique uses an intensity modulated laser to create periodically changing temperature in a sample that is probed by thermoreflectance. ${ }^{16,24}$ The phase lag of temperature, with respect to heat flux is fit with an analytical heat conduction model to extract the unknown TBR. Here, we focused the laser directly onto the 3-omega patterns of the $100 \mathrm{~nm}$ AIN film. High frequency modulation $(0.1-20 \mathrm{MHz}$, compared to $0.3-$ $3000 \mathrm{kHz}$ for 3-omega) and the deeply buried CMP-SiC/AlN 

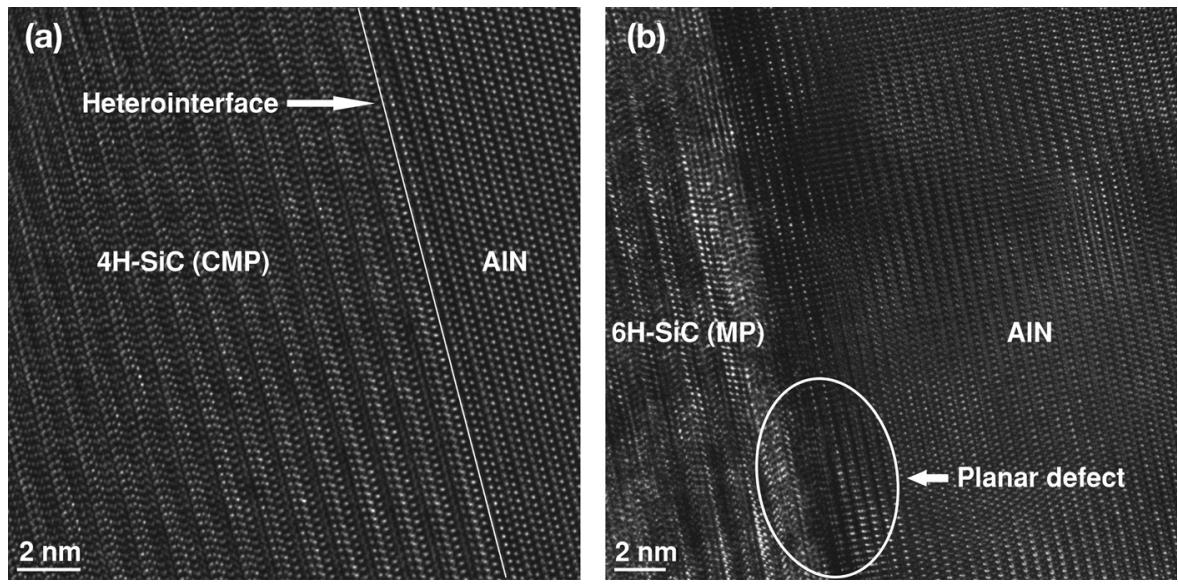

FIG. 3. Comparison of the interfaces between AlN/CMP SiC and AlN/MP SiC. (a) AlN grown on $\mathrm{CMP} 4 \mathrm{H}-\mathrm{SiC}(0001)$ has a clearly defined interface with no apparent additional strain in the film. (b) By contrast, microstructural roughness of the surface of the MP $6 \mathrm{H}-\mathrm{SiC}(0001)$ generated stresses and resultant strains that caused planar defects within the first few atomic layers of the AlN film. The periodicity of the AlN is recovered within a few nm of the interface in both films, supporting our hypothesis that interface defects contribute to the thermal boundary resistance of the heterostructures.

TABLE II. Thermal Boundary Resistance of the AlN film with the $\mathrm{SiC}$ substrates and $\mathrm{Ti}$ or $\mathrm{SiO}_{2}$ top layers. The TBR of the higher roughness MP-SiC substrate is more than an order of magnitude larger than the TBR of the CMP-SiC substrate.

\begin{tabular}{llcc}
\hline \hline Heterostructure (Sub/AlN/top) & \multicolumn{1}{c}{$\mathrm{TBR}_{\text {sub }}+\mathrm{TBR}_{\text {top }}\left(\mathrm{m}^{2} \mathrm{~K} / \mathrm{GW}\right)$} & $\mathrm{TBR}_{\text {sub }}\left(\mathrm{m}^{2} \mathrm{~K} / \mathrm{GW}\right)$ & $\mathrm{TBR}_{\text {top }}\left(\mathrm{m}^{2} \mathrm{~K} / \mathrm{GW}\right)$ \\
\hline $\mathrm{CMP}-\mathrm{SiC} / \mathrm{AlN} / \mathrm{Ti}$ & $\mathrm{TBR}_{\mathrm{CMP}-\mathrm{SiC} / \mathrm{AlN}}+\mathrm{TBR}_{\mathrm{Ti} / \mathrm{AlN}}=13 \pm 2^{\mathrm{a}}$ & $5.1 \pm 2.8$ & $7.9 \pm 2.0^{\mathrm{b}}$ \\
$\mathrm{CMP}-\mathrm{SiC} / \mathrm{AlN} / \mathrm{SiO}_{2}$ & $\mathrm{TBR}_{\mathrm{CMP}-\mathrm{SiC} / \mathrm{AlN}}+\mathrm{TBR}_{\mathrm{SiO} / \mathrm{AlN}}=31 \pm 5^{\mathrm{a}}$ & $5.1 \pm 2.8$ & $25.9 \pm 5.7$ \\
$\mathrm{MP}-\mathrm{SiC} / \mathrm{AlN} / \mathrm{SiO}_{2}$ & $\mathrm{TBR}_{\mathrm{MP}-\mathrm{SiC} / \mathrm{AlN}}+\mathrm{TBR}_{\mathrm{SiO} / \mathrm{AlN}}=120 \pm 20^{\mathrm{a}}$ & $94 \pm 21$ & $25.9 \pm 5.7$ \\
\hline \hline
\end{tabular}

${ }^{a}$ Measured by 3 -omega.

${ }^{\mathrm{b}}$ Measured by FDTR.

interface made this measurement most sensitive to the Ti/ AlN interface. FDTR found $T B R_{\mathrm{Ti} / \mathrm{AlN}}=7.9 \pm 2.0 \mathrm{~m}^{2} \mathrm{~K} / \mathrm{GW}$ (see supplementary online material ${ }^{18}$ for FDTR data and fit). This value is comparable but higher than prior measurements of $\mathrm{Cr} / \mathrm{AlN}$ interfaces $\left(5 \mathrm{~m}^{2} \mathrm{~K} / \mathrm{GW}\right),{ }^{25}$ conceivably due to increased mismatch in the Debye temperatures $\theta_{\mathrm{D}}$ of $\mathrm{AlN}$ $\left(\theta_{\mathrm{D}-\mathrm{AlN}}=950 \mathrm{~K}\right)$ and $\mathrm{Ti}\left(\theta_{\mathrm{D}-\mathrm{Ti}}=420 \mathrm{~K}\right)$, relative to AlN and $\mathrm{Cr}\left(\theta_{\mathrm{D}-\mathrm{Cr}}=630 \mathrm{~K}\right) .^{26}$

The value of $T B R_{\mathrm{Ti} / \mathrm{AlN}}$ was used to calculate the remaining unknown TBRs, which are listed in Table II. Uncertainty estimates are based on propagation of measurement uncertainty. Notably, the value of $T B R_{\mathrm{AlN} / \mathrm{MP}-\mathrm{SiC}}$ is more than an order of magnitude larger than the value of

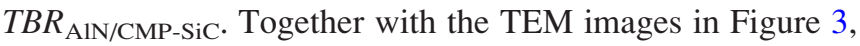
this confirms that substrate roughness degrades the interface structure and phonon transport properties, as also observed in $\mathrm{GaSb} / \mathrm{GaAs}$ and $\mathrm{Al} / \mathrm{Si}$ interfaces. ${ }^{13,14}$ Prior measurements of amorphous/crystalline interfaces such as $\mathrm{SiO}_{2} / \mathrm{Si}$ and $\mathrm{SiN}_{\mathrm{x}} / \mathrm{Si}$ identify TBRs of $20 \mathrm{~m}^{2} \mathrm{~K} / \mathrm{GW}$ at $T=300 \mathrm{~K}^{17}$ that are comparable to $T B R_{\mathrm{SiO} / \mathrm{AlN}}$.

Though LED manufacturers have moved towards CMP$\mathrm{SiC}$, our data suggest that surface roughness impacts the interface and thermal transport properties more profoundly than different AIN growth methods and substrate materials. Accordingly, the value of $k_{\text {AlN-eff }}=0.93 \pm 0.16 \mathrm{~W} / \mathrm{mK}$ reported in our prior study should be used for with MP$\mathrm{SiC},{ }^{10}$ while $k_{\text {AlN-eff }}=6-7 \mathrm{~W} / \mathrm{mK}$ should be used for CMP$\mathrm{SiC}$ based devices in predicting operating temperature. For the CMP-SiC/AlN interface, we find a TBR of $5.1 \pm 2.8$ $\mathrm{m}^{2} \mathrm{~K} / \mathrm{GW}$, which is equivalent to roughly $240 \mathrm{~nm}$ of highly dislocated AlN $\left(k_{\mathrm{AlN}}=47 \mathrm{~W} / \mathrm{mK}\right)$ or $1450 \mathrm{~nm}$ of single crystal AlN $\left(k_{\text {AlN-Slack }}=285 \mathrm{~W} / \mathrm{mK}^{5}\right)$ at $T=300 \mathrm{~K}$. This value is comparable to the total AIN film resistance measured by Cho et al.. ${ }^{12}$ for very thin films $(\sim 30 \mathrm{~nm})$ as well as the lowest values measured on a range of AlN nucleation layers by Manoi et al. ${ }^{2}$ Our study suggests that differences result primarily from the microstructure of the AIN near its interface with $\mathrm{SiC}$. The diffuse mismatch model predicts that the TBR of a perfect AlN-SiC interface can be as low as $0.6 \mathrm{~m}^{2} \mathrm{~K} /$ GW. ${ }^{12}$ To the detriment of heat dissipation in many important technologies, the defects near the AlN-SiC interface clearly play a significant role in increasing the TBR beyond this value.

The work was supported in part by NSF Grant No. 1133394 and the Dowd-ICES Fellowship at Carnegie Mellon University. The authors express their appreciation to Cree, Inc. for providing the $\mathrm{SiC}$ substrates.

1"Thermal management of white LEDs," PNNL-SA-51901, Building Technologies Program, EERE, DOE (2009).

${ }^{2}$ A. Manoi, J. W. Pomeroy, N. Killat, and M. Kuball, IEEE Electron Device Lett. 31, 1395-1397 (2010).

${ }^{3}$ G. J. Riedel, J. W. Pomeroy, K. P. Hilton, J. O. Maclean, D. J. Wallis, M. J. Uren, T. Martin, U. Forsberg, A. Lundskog, A. Kakanakova-Georgieva, G. Pozina, E. Janzen, R. Lossy, R. Pazirandeh, F. Brunner, J. Wurfl, and M. Kuball, IEEE Electron Device Lett. 30, 103-106 (2009).

${ }^{4}$ W. L. Liu and A. A. Balandin, Appl. Phys. Lett. 85, 5230-5232 (2004).

${ }^{5}$ G. A. Slack, R. A. Tanzilli, R. O. Pohl, and J. W. Vandersande, J. Phys. Chem. Solids 48, 641-647 (1987).

${ }^{6}$ Y. K. Koh, Y. Cao, D. G. Cahill, and D. Jena, Adv. Funct. Mater. 19, 610-615 (2009).

${ }^{7}$ M. Dinescu, A. Jacquot, B. Lenoir, A. Dauscher, P. Verardi, F. Craciun, M. Stolzer, and M. Gartner, Appl. Surf. Sci. 186, 507-512 (2002).

${ }^{8}$ Z. J. Reitmeier, S. Einfeldt, R. F. Davis, X. Y. Zhang, X. L. Fang, and S. Mahajan, Acta Mater. 57, 4001-4008 (2009). 
${ }^{9}$ Z. J. Reitmeier, S. Einfeldt, R. F. Davis, X. Y. Zhang, X. L. Fang, and S. Mahajan, Acta Mater. 58, 2165-2175 (2010).

${ }^{10}$ Z. Su, L. Huang, F. Liu, J. P. Freedman, L. M. Porter, R. F. Davis, and J. A. Malen, Appl. Phys. Lett. 100, 201106 (2012).

${ }^{11}$ A. Sarua, H. Ji, K. P. Hilton, D. J. Wallis, M. J. Uren, T. Martin, and M. Kuball, IEEE Trans. Electron Devices 54, 3152-3158 (2007).

${ }^{12}$ J. W. Cho, E. Bozorg-Grayeli, D. H. Altman, M. Asheghi, and K. E. Goodson, IEEE Electron Device Lett. 33, 378-380 (2012).

${ }^{13}$ P. E. Hopkins, J. C. Duda, S. P. Clark, C. P. Hains, T. J. Rotter, L. M. Phinney, and G. Balakrishnan, Appl. Phys. Lett. 98, 161913 (2011).

${ }^{14}$ P. E. Hopkins, L. M. Phinney, J. R. Serrano, and T. E. Beechem, Phys. Rev. B 82, 085307 (2010).

${ }^{15}$ D. G. Cahill, Rev. Sci. Instrum. 61, 802-808 (1990).

${ }^{16}$ J. A. Malen, K. Baheti, T. Tong, Y. Zhao, J. A. Hudgings, and A. Majumdar, ASME J. Heat Transfer 133, 081601 (2011).
${ }^{17}$ S. M. Lee and D. G. Cahill, J. Appl. Phys. 81, 2590-2595 (1997).

${ }^{18}$ See supplementary material at http://dx.doi.org/10.1063/1.4808238 for AlN growth details; for TEM imaging details; for 3-omega Method details; and for frequency domain thermoreflectance (FDTR) details and data.

${ }^{19}$ J. Callaway, Phys. Rev. 113, 1046-1051 (1959).

${ }^{20}$ F. Yang and C. Dames, Phys. Rev. B 87, 035437 (2013).

${ }^{21}$ G. Chen and C. L. Tien, J. Thermophys. Heat Transfer 7, 311-318 (1993).

${ }^{22}$ A. Majumdar, ASME J. Heat Transfer 115, 7-16 (1993).

${ }^{23}$ K. E. Goodson, O. W. Kading, M. Rosler, and R. Zachai, J. Appl. Phys. 77, 1385-1392 (1995).

${ }^{24}$ A. J. Schmidt, R. Cheaito, and M. Chiesa, Rev. Sci. Instrum. 80, 094901 (2009).

${ }^{25}$ R. J. Stevens, A. N. Smith, and P. M. Norris, ASME J. Heat Transfer 127, 315-322 (2005).

${ }^{26}$ C. Kittel, Introduction to Solid State Physics, 8th ed. (Wiley, Hoboken, NJ, 2005) 